A composite dislocation cell model to describe strain path change effects in BCC metals

This article has been downloaded from IOPscience. Please scroll down to see the full text article.

2009 Modelling Simul. Mater. Sci. Eng. 17064008

(http://iopscience.iop.org/0965-0393/17/6/064008)

View the table of contents for this issue, or go to the journal homepage for more

Download details:

IP Address: 131.180.130.109

The article was downloaded on 08/08/2011 at 09:59

Please note that terms and conditions apply. 


\title{
A composite dislocation cell model to describe strain path change effects in $\mathrm{BCC}$ metals
}

\author{
T Yalcinkaya ${ }^{1,2}$, W A M Brekelmans ${ }^{2}$ and M G D Geers ${ }^{2}$ \\ ${ }^{1}$ Materials Innovation Institute M2I, PO Box 5008, 2600 GA Delft, The Netherlands \\ 2 Department of Mechanical Engineering, Eindhoven University of Technology, PO Box 513, \\ $5600 \mathrm{MB}$ Eindhoven, The Netherlands \\ E-mail: t.yalcinkaya@tue.nl
}

Received 26 February 2009, in final form 26 June 2009

Published 24 August 2009

Online at stacks.iop.org/MSMSE/17/064008

\begin{abstract}
Sheet metal forming processes are within the core of many modern manufacturing technologies, as applied in, e.g., automotive and packaging industries. Initially flat sheet material is forced to transform plastically into a three-dimensional shape through complex loading modes. Deviation from a proportional strain path is associated with hardening or softening of the material due to the induced plastic anisotropy resulting from the prior deformation. The main cause of these transient anisotropic effects at moderate strains is attributed to the evolving underlying dislocation microstructures. In this paper, a composite dislocation cell model, which explicitly describes the dislocation structure evolution, is combined with a BCC crystal plasticity framework to bridge the microstructure evolution and its macroscopic anisotropic effects. Monotonic and multi-stage loading simulations are conducted for a single crystal and polycrystal BCC metal, and the obtained macroscopic results and dislocation substructure evolution are compared qualitatively with the published experimental observations.
\end{abstract}

\section{Introduction}

For each car, the automotive industry manufactures more than 500 parts by multi-stage forming operations, involving complex deformation paths. Deviation from a proportional strain path is commonly associated with a change in the hardening (or softening) behavior of the material. In order to achieve a first-time-right design, modern predictive tools relying on the finite element method are commonly used nowadays. The anisotropy induced by complex deformation paths, which may lead to premature failure (e.g. Sang and Lloyd (1979)), is crucial in this sense and should be included in the constitutive models used in the analysis.

The overall plastic anisotropy in BCC metals, induced by the imposed deformation, originates from different sources at different length scales. Slip asymmetry and intrinsic anisotropy effects caused by the non-planar spreading of screw dislocation cores are active at 
the microlevel (e.g. Bassani et al (2001), Duesbery and Vitek (1998), Ito and Vitek (2001), Yalcinkaya et al (2008)) whereas the development of dislocation substructures is relevant at the mesolevel (e.g. Rauch and Schmitt (1989), Wagoner and Laukonis (1983), Rao and Laukonis (1983), Wilson and Bate (1994), Gardey et al (2005)). At the macrolevel, the texture development of polycrystalline metal contributes dominantly (e.g. Bacroix et al (1994), Bacroix and Hu (1995), Nesterova et al (2001)). Upon switching strain paths, the intrinsic properties obviously have a substantial effect on the observed anisotropy due to changes in the dislocation activity. However, the evolution of dislocation microstructures has been recognized as a main driver triggering the observed anisotropic material behavior. In a recent report, Li et al (2006) commented on the strong anisotropy, i.e. larger than expected from the texture, induced by the dislocation structure in IF steel increasing with the rolling prestrain. The prediction of dislocation microstructures within the individual dislocation descriptions and continuum theories has been a challenging subject in the last decades in the material science community (see Groma (1997) for an overview). While transmission electron microscopy (TEM) observations have been a powerful tool to understand their origin and to derive the physical parameters that govern their evolution (e.g. Fernandes and Schmitt (1983)), discrete dislocation models and atomistic considerations improved the understanding of the formation and the evolution of dislocation microstructures and the related plastic anisotropy. However, only a limited number of micromechanical modeling approaches have been addressing the anisotropy induced by evolving dislocation cells with a crystal plasticity framework.

Among the attempts to develop plastic anisotropy models that incorporate the microstructure evolution for complex deformation histories, the most remarkable one is the constitutive model proposed by Teodosiu and $\mathrm{Hu}$ (1995). This phenomenological model uses the Hill criterion for the onset of yielding while the hardening is associated with the dislocation structures. The polarity of dislocation walls, the back-stress and the strength of the dislocation structure are accounted for by internal variables. Recently, Wang et al (2008) presented an improvement of this model especially concentrating on continuous loading path changes from uniaxial tension to simple shear without unloading the material.

Another attempt to describe the occurring phenomena is presented by Peeters et al (2000) dealing with a polycrystal plasticity model that incorporates more details of the microstructure evolution at the grain scale, where cell boundary dislocation densities, cell block boundary dislocation densities and directionally movable dislocation densities are taken as internal variables. This model attributes a major part of the strain path change effects to the evolution of cell block boundaries and the polarization of these structures. Additional to the above mentioned models, Hoc and Forest (2001), Mollica et al (2001) and Tarigopula et al (2008) presented some other approaches dealing with the anisotropic strain path change effects. In this paper the concentration is focused on a crystal plasticity model that incorporates the evolution of dislocation cell structures. As originally introduced by Mughrabi (1987), a cell structure can be idealized as a two-component material, distinguishing cell walls and cell interiors. It is characterized by the wall thickness $w$, the cell size $r$ (see figure 1), the dislocation densities in the cell walls $\rho^{\mathrm{w}}$ and the cell interiors $\rho^{\mathrm{c}}$. The macroscopic anisotropy effects are obtained by the evolution of these internal variables during monotonic deformation and multi-stage loading processes. Inside the cell structure, a BCC crystal plasticity framework (e.g. Yalcinkaya et al (2008)) is incorporated, which goes beyond the developments of Viatkina et al (2003) for FCC metals in which a classical von-Mises plasticity model was used. From this perspective, it is the first example that incorporates a physically motivated constitutive model into the evolution of dislocation substructures for BCC metals, in order to model the anisotropy due to strain path changes. 


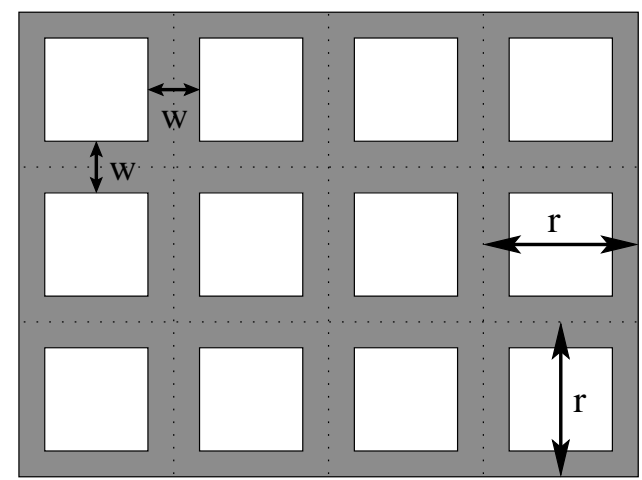

Figure 1. Composite representation of the cell structure.

The plan of this paper is as follows. Section 2 discusses the evolution of dislocation substructures under monotonic and multi-stage deformations. Next, in section 3 the formulation of the BCC crystal plasticity framework is summarized. Section 4 handles the incorporation of the dislocation cell evolution model into the crystal plasticity framework, along with a summary of the numerical implementation. Further, in section 5 computational results of single crystal and polycrystal tests are presented on the basis of which the crystal anisotropy is distinguished from the dislocation cell anisotropy. The accordance of the results with respect to published experimental results is discussed. Finally, concluding remarks are given in section 6 .

Cartesian tensors and associated tensor products will be used throughout this paper, making use of a Cartesian vector basis $\left\{\boldsymbol{e}_{1} \boldsymbol{e}_{2} \boldsymbol{e}_{3}\right\}$. Using the Einstein summation rule for repeated indices, the following conventions are used in the notations of vectors, tensors, related products and crystallography:

- scalars $a$

- vectors $\boldsymbol{a}=a_{i} \boldsymbol{e}_{i}$

- second-order tensors $\boldsymbol{A}=A_{i j} \boldsymbol{e}_{i} \otimes \boldsymbol{e}_{j}$

- fourth-order tensors ${ }^{4} \boldsymbol{A}=A_{i j k l} e_{i} \otimes e_{j} \otimes e_{k} \otimes e_{l}$

- $\boldsymbol{C}=\boldsymbol{a} \otimes \boldsymbol{b}=a_{i} b_{j} \boldsymbol{e}_{i} \otimes \boldsymbol{e}_{j}$

- $\boldsymbol{C}=\boldsymbol{A} \cdot \boldsymbol{B}=A_{i j} B_{j k} \boldsymbol{e}_{i} \otimes \boldsymbol{e}_{k}$

- $\boldsymbol{C}={ }^{4} \boldsymbol{A}: \boldsymbol{B}=A_{i j k l} B_{l k} \boldsymbol{e}_{i} \otimes \boldsymbol{e}_{j}$

- crystallographic direction, family [uvw], $\langle u v w\rangle$

- crystallographic plane, family $(h k l),\{h k l\}$

- slip system, family $(h k l)[u v w],\{h k l\}\langle u v w\rangle$

\section{Dislocation substructure evolution}

Dislocation substructuring is characterized by the clustering of dislocations after a certain amount of plastic deformation, where an initially statistically homogeneous distribution of dislocations develops towards a dislocation pattern with high density dislocation walls enveloping low density dislocation areas. This self-organization of the microstructure in the grains is often referred to as the low-energy, steady state configuration of dislocations (Kuhlmann-Wilsdorf 1989). TEM analyses (e.g. Keh et al (1963)) revealed that for deformations larger than 3-4\% a well-developed dislocation cell structure forms in steel at 


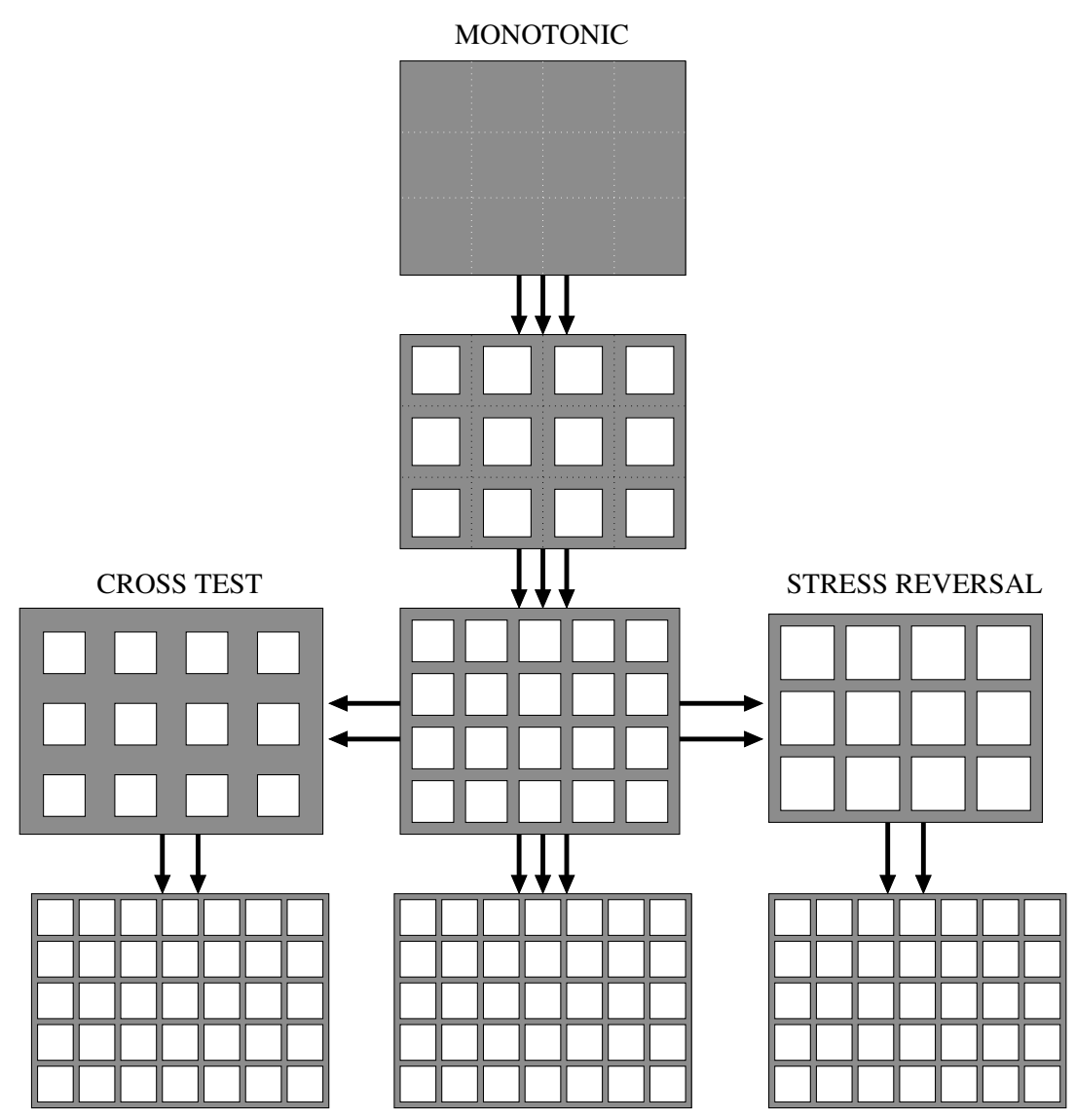

Figure 2. Schematic evolution of a dislocation cell structure under strain path change.

ambient temperature. Further deformation renders a polarized structure with dislocation sheets or cell block boundaries, which envelope a number of dislocation cells. These structures are the result of the interactions between dislocations gliding on the most active slip planes and the secondary dislocations (Teodosiu 1992). However, the occurrence of the dislocation sheets is not always manifest and sometimes the microstructure is partitioned by ordinary cell boundaries having no particular crystallographic or macroscopic orientation (Hansen and Huang 1997). For that reason, depending on the grain orientation and the strain direction, either parallel dislocation walls or more equiaxed closed cells are observed (e.g. Rauch and Schmitt (1989)) in low-carbon steels. Besides, different materials end up in different types of microstructures. It is neither experimentally nor computationally an easy task to identify the type of evolving dislocation microstructure, yet formation of dislocation cells is mostly observed. Thereof, this paper concentrates on the formation and evolution of these dislocation cell structures.

As discussed above, dislocation cell structures develop upon plastic strain in most metals, and evolve in a distinct way depending on the applied strain path. The main features are visualized in figure 2. Under monotonic deformation a dislocation cell structure appears and evolves towards a decreasing cell size $r$, and wall thickness $w$ accompanied by an increasing dislocation density in the cell walls $\rho^{\mathrm{w}}$ (e.g. Fernandes and Schmitt (1983)). After a strain path change, the developed cell structure adjusts to the new loading and the dislocation 
microstructure induced by the prestrain becomes unstable. It is disrupted and dissolved, and a new dislocation structure typical of the new strain path forms (Barlat et al 2003). The characteristic features of the initial cell structure disappear as the deformation proceeds in the new direction. Unfortunately, there is no clear interpretation of what is occurring with the dislocation microstructure during the adaptation nor is there a unique terminology to describe this evolution. Here we distinguish between two different scenarios; dissolution of cells as in the cross test and disruption as occurring under reversed loading. There appears to be no consistency in the literature in the use of the dissolution, disruption and disintegration of dislocation cells, and most of the time any cell evolution after a strain path change is described as a dissolution process (e.g. Rauch and Schmitt (1989), Rauch (1992), Rauch (1991), Gardey et al (2005), Rao and Laukonis (1983)). Indeed, both dissolved and disrupted structures appear as disorganized structures with a higher degree of homogeneity compared with the state before the strain path change. Nevertheless, there are indications that there is a morphological difference between the two microstructure evolution scenarios mentioned above (e.g. Gardey et al (2005)) in correspondence with the difference between the driving forces and their physical origins.

In the example shown in figure 2, two different strain path changes have been considered where the two types of evolution phenomena can be distinguished. A cross test, e.g. tension followed by simple shear or a tension test followed by tension in a different direction, reveals progressive cell evolution (e.g. Rao and Laukonis (1983)). It has been observed that after a strain path change cell walls become thicker while the dislocation density in the walls becomes smaller (e.g. Schmitt et al (1991)). Hence, the dislocation distribution is more uniform and the cell structure is less organized. This evolution process can cause partial or complete dissolution of the existing cell structure, while concurrently a new cell structure develops with a morphology related to the new loading direction. The cell structure evolution resulting from a stress reversal has received more attention in the context of the analysis of the well-known Bauschinger effect (e.g. Rauch (1991)). The evolution of the cell structure under stress reversal can be characterized by the disruption of cell walls (e.g. Viatkina (2005), Christodoulou et al (1986)). The thickness of the cell walls does not change significantly; however, the walls tend to disconnect. Experimental observations (e.g. Christodoulou et al (1986)) also report a strong flux of dislocations from the walls to the cell interiors, decreasing the wall dislocation density and increasing the density in the cell interiors. Accordingly, the descriptive modeling of the dislocation distribution relies on an increase in cell size and a dislocation redistribution (Viatkina 2005). With ongoing deformation cell walls reappear and a new cell structure originates.

Upon sustained loading after a strain path change, the microstructure always evolves such that transient effects disappear and the macroscopic stress-strain curve saturates to the monotonic deformation curve (see figure 3 ).

\section{Computational model}

The constitutive behavior of each composite constituent (cell walls or cell interiors) is modeled in a finite strain crystal plasticity framework with plastic slip governed by the thermally activated motion of dislocations. The kinematics starts with the multiplicative decomposition of the deformation gradient tensor into an elastic and a plastic part of each component, as developed by Lee (1969), Rice (1971), Hill and Rice (1972) in the classical plasticity theory,

$$
\boldsymbol{F}^{i}=\boldsymbol{F}_{\mathrm{e}}^{i} \cdot \boldsymbol{F}_{\mathrm{p}}^{i}
$$



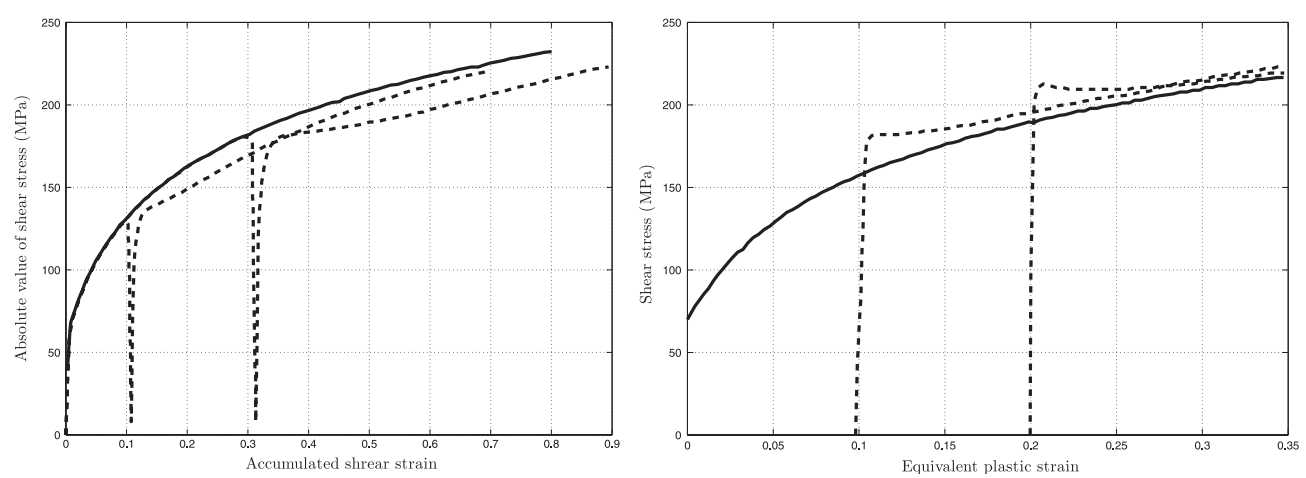

Figure 3. Left: Experimental results for mild steel DC06 subjected to monotonic simple shear and simple shear followed by load reversal (10\% and 30\%) (Bouvier et al 2006) (reversed loading) Right: experimental results for IF-steel subjected to monotonic simple shear and tensile tests (10\% and 20\%) followed by shear (Peeters et al 2000) (orthogonal loading).

where the superscript $i$ indicates the specific component (w: wall, c: cell) and tensor $\boldsymbol{F}_{\mathrm{p}}^{i}$ defines the stress-free intermediate configuration. In this configuration, resulting from plastic shearing along well-defined slip planes of the crystal lattice, the orientation of the slip systems is unaltered. The tensor $\boldsymbol{F}_{\mathrm{e}}^{i}$ reflects the lattice deformation and local rigid body rotations. The slip systems are labeled by a superscript $\alpha$, with $\alpha=1,2, \ldots, n_{\mathrm{s}}$ where $n_{\mathrm{s}}$ is the total number of slip systems. The vectors $\boldsymbol{m}_{0}^{\alpha, i}$ and $\boldsymbol{n}_{0}^{\alpha, i}$ denote the slip direction and the slip plane normal in the reference and intermediate configurations. In the current state they are represented by $\boldsymbol{m}^{\alpha, i}$ and $\boldsymbol{n}^{\alpha, i}$, respectively.

The crystallographic split of the plastic flow rate $\boldsymbol{L}_{\mathrm{p}}^{i}=\dot{\boldsymbol{F}}_{\mathrm{p}}^{i} \cdot \boldsymbol{F}_{\mathrm{p}}^{i-1}$ is given by

$$
\boldsymbol{L}_{\mathrm{p}}^{i}=\sum_{\alpha=1}^{n_{s}} \dot{\gamma}^{\alpha, i} \boldsymbol{m}_{0}^{\alpha, i} \otimes \boldsymbol{n}_{0}^{\alpha, i},
$$

with $\dot{\gamma}^{\alpha, i}$ the individual slip rate on the slip system $\alpha$.

The second Piola-Kirchhoff stress tensor $\boldsymbol{S}^{i}$ is expressed in terms of the elastic GreenLagrange strain tensor $\boldsymbol{E}_{\mathrm{e}}^{i}$, both relative to the intermediate state,

$$
\boldsymbol{S}^{i}={ }^{4} \boldsymbol{C}: \boldsymbol{E}_{\mathrm{e}}^{i} \quad \text { with } \quad \boldsymbol{E}_{\mathrm{e}}^{i}=\frac{1}{2}\left(\boldsymbol{F}_{\mathrm{e}}^{i^{\mathrm{T}}} \cdot \boldsymbol{F}_{\mathrm{e}}^{i}-\boldsymbol{I}\right),
$$

with $\boldsymbol{I}$ the second-order unity tensor and ${ }^{4} \boldsymbol{C}$ the fourth order tensor consisting of elastic moduli.

From the second Piola-Kirchhoff stress the Kirchhoff stress in the current configuration can be determined by a push-forward operation,

$$
\tau^{i}=F_{\mathrm{e}}^{i} \cdot S^{i} \cdot F_{\mathrm{e}}^{i^{\mathrm{T}}}
$$

From the Kirchhoff stress the Cauchy stress can be derived according to

$$
\boldsymbol{\sigma}^{i}=\frac{1}{J_{\mathrm{e}}^{i}} \tau^{i} \quad \text { with } \quad J_{\mathrm{e}}^{i}=\operatorname{det}\left(\boldsymbol{F}_{\mathrm{e}}^{i}\right) .
$$

The Schmid resolved shear stress is the projection of the Kirchhoff stress on the slip systems, i.e.

$\tau^{\alpha, i}=\boldsymbol{m}^{\alpha, i} \cdot \boldsymbol{\tau}^{i} \cdot \boldsymbol{n}^{\alpha, i}=\boldsymbol{m}_{0}^{\alpha, i} \cdot \boldsymbol{C}_{\mathrm{e}}^{i} \cdot \boldsymbol{S}^{i} \cdot \boldsymbol{n}_{0}^{\alpha, i} \quad$ with $\quad \boldsymbol{C}_{\mathrm{e}}^{i}=\boldsymbol{F}_{\mathrm{e}}^{i^{\mathrm{T}}} \cdot \boldsymbol{F}_{\mathrm{e}}^{i}$

which is the driving force for the dislocation movement on a certain slip system $\alpha$. There has been various discussions and contradictions considering the active slip systems of BCC crystals, and recent studies show that the slip system activation is highly temperature dependent (see 
Yalcinkaya et al (2008)). At room temperature the $\left\{\begin{array}{lll}1 & 1 & 2\end{array}\right\}$ slip system family is dominantly active. The effect of the non-Schmid stresses on the non-planar screw dislocation cores which contributes to the orientation dependence of BCC crystals at the single crystal level can be taken into account as an additional contribution to the driving stress in equation (6) (Yalcinkaya et al 2008); however, this contribution affects the initial anisotropy of these metals rather than the transient effects observed during the strain path changes (see figure 3 ). Including this effect would increase the material parameters while it does not contribute to the aim of this paper. Hence it was decided not to account for this effect here.

The motion of dislocations is obstructed by thermal and a-thermal barriers which are caused by the dislocation interactions upon flow, the elastic stress field due to other dislocations and grain boundaries. Hence the slip resistance distinguishably originates from a thermal part $s_{\mathrm{t}}^{\alpha, i}$ and an a-thermal part $s_{a}^{\alpha, i}$. For the slip rates the following slip law is adopted (see Yalcinkaya et al (2008)):

$$
\dot{\gamma}^{\alpha, i}=\dot{\gamma}_{0}^{\alpha, i} \exp \left\{\frac{-G_{0}}{k T}\left[1-\left(\frac{\tau_{\mathrm{eff}}^{\alpha, i}}{s_{\mathrm{t}}^{\alpha, i}}\right)\right]\right\} \operatorname{sign}\left(\tau^{\alpha, i}\right),
$$

where $\tau_{\text {eff }}^{\alpha, i}=\left|\tau^{\alpha, i}\right|-s_{a}^{\alpha, i}$ is the effective driving stress on the slip systems, $G_{0}$ is the activation free energy, $k$ is Boltzmann's constant, $T$ is the absolute temperature and $\dot{\gamma}_{0}^{\alpha, i}$ is a reference strain rate. For isothermal cases, the thermal part $s_{\mathrm{t}}^{\alpha, i}$ of the slip resistance is taken constant and the a-thermal slip resistance is related to the dislocation densities on all slip systems through

$$
s_{a}^{\alpha, i}=G b \sqrt{\sum_{u=1}^{n_{\mathrm{s}}} A^{\alpha u}\left|\rho^{u, i}\right|,}
$$

where $G$ is the shear modulus, $b$ is the magnitude of the Burgers vector, $A^{\alpha u}$ are the interaction coefficients between the slip systems $\alpha$ and $u$ and $\rho^{u, i}$ the dislocation density on the slip system $u$ of component $i$. The dislocation interaction coefficients of the matrix $A^{\alpha u}$ depend on the type of interaction between dislocations on different slip systems (e.g. Franciosi and Zaoui (1982), Queyreau et al (2008)). Because of the lack of data on $\left\{\begin{array}{lll}1 & 1 & 2\end{array}\right\}$ slip systems, only the interactions between the dislocations belonging to the same slip system, i.e. $\alpha=u$, and different slip systems, i.e. $\alpha \neq u$, will be distinguished for $A^{\alpha u}$.

The macroscopic mechanical response of the composite model is obtained by applying a Taylor averaging assumption where the deformation in each component is assumed to be equal to the macroscopic deformation and where the rule of mixtures gives the macroscopic stress from the local stresses in each component according to

$$
\boldsymbol{\sigma}=f \boldsymbol{\sigma}^{\mathrm{w}}+(1-f) \boldsymbol{\sigma}^{\mathrm{c}} \text {. }
$$

In this equation $f$ represents the actual volume fraction of the cell walls, expressed in terms of the microstructural morphology parameters $w$ and $r$ according to

$$
f=\frac{V^{\mathrm{w}}}{V}=3 \frac{w}{r}-3\left(\frac{w}{r}\right)^{2}+\left(\frac{w}{r}\right)^{3},
$$

where $V$ and $V^{\mathrm{w}}$ are the volumes of the entire composite and the wall component, respectively.

Experimental studies (e.g. Fernandes and Schmitt (1983)) suggest that the wall thickness $w$, the cell size $r$ (see figure 1), the dislocation densities in the cells $\rho^{\alpha, \mathrm{c}}$, and the walls $\rho^{\alpha, w}$ evolve with increasing applied strain. Moreover, these quantities are dependent on the deformation history, and therefore they are taken into account as internal variables in this framework. Corresponding evolution equations are to be formulated that describe the cell structure development during monotonic loading as well as complex strain path histories. This is done in the following section, where the incorporation of a dislocation cell structure evolution model into the crystal plasticity framework is presented. 


\section{Modeling of microstructure evolution}

In order to give a clear understanding of the model, three distinct types of loading cases are considered, namely monotonic loading, orthogonal loading and reverse loading. The purpose of the model consists in unifying these cases by capturing effects under continuous combinations of these deformations, through a single set of evolution equations. It is assumed that the cell orientation is dictated by the loading, and that there are always enough slip systems to accommodate that cell, independently of the crystal orientation.

\subsection{Monotonic deformation}

The evolution of a two-phase dislocation cell structure has been schematized in figure 2. During monotonic deformation, the cell size $r$ and the wall thickness $w$ decrease, yielding a decrease in the length scales of the spatial dislocation patterns that is inversely proportional to the flow stress, often referred to as the law of similitude (Kuhlmann-Wilsdorf 1962). Experimental observations (e.g. Mughrabi et al (1986)) suggest that the dislocation density inside the cell interiors does not change significantly. Accordingly, a constant dislocation density $\rho^{\alpha, \mathrm{c}}$ in the cell interior component is assumed here. The derivation of the evolution of the dislocation density in the walls $\rho^{\alpha, \mathrm{w}}$ departs from the frequently used balance between the multiplication of mobile dislocations and annihilation events,

$$
\dot{\rho}^{\alpha, \mathrm{w}}=\frac{1}{b}\left[I \sqrt{\rho^{\alpha, \mathrm{w}}}-R \rho^{\alpha, \mathrm{w}}\right] \sum_{\alpha}\left|\dot{\gamma}^{\alpha, \mathrm{w}}\right|+\frac{\rho^{\alpha, \mathrm{c}}-\rho^{\alpha, \mathrm{w}}}{f} \dot{f}
$$

where $R$ is the recovery length and $I$ is a dislocation multiplication parameter. The last term in the equation accounts for the change in volume occupied by the wall component. The initial state of the composite is modeled as if the wall component is occupying the entire volume; the initial value of its dislocation densities is determined by the value $\rho_{0}$ of the initial uniform distribution. This in fact correctly represents the case when no dislocation pattern is present.

The following empirical relation between the cell size $r$ and the flow stress $\sigma^{y}$ is suggested in the literature (e.g. Barker et al (1989), Mughrabi (1987)),

$$
\sigma^{y}=\frac{C G b}{r^{m}}
$$

which is consistent with the experimental observations of Fernandes and Schmitt (1983) and commonly used theoretical investigations (e.g. Mughrabi (1987)). The parameter $C$ is a material constant and the exponent $m$ is generally close to 1 for cell structures. In this framework, equation (12) is rewritten in terms of slip variables at the slip system level instead of the continuum level yield stress $\sigma^{y}$. This is done by using evolving a-thermal slip resistances on the active slip systems, i.e. $r \sim C G b / \sigma^{y} \sim C G b / \sum_{\alpha} s_{a}$, whereby the parameter $C$ accounts for the scaling between the two levels as well. The cell size evolution is approximated by incorporating the rule of mixtures for the different components of the composite,

$$
r=\frac{C G b}{f \sum_{\alpha} s_{a}^{\alpha, \mathrm{w}}+(1-f) \sum_{\alpha} s_{a}^{\alpha, \mathrm{c}}} .
$$

The evolution of the wall thickness $w$ is adopted from Viatkina et al (2003) and assumed to be governed by an effective plastic strain rate measure $\sum_{\alpha}\left|\dot{\gamma}^{\alpha}\right|$ according to

$$
\dot{w}=k_{\mathrm{m}}\left(w_{\text {inf }}-w\right) \sum_{\alpha}\left|\dot{\gamma}^{\alpha}\right|
$$

with

$$
\sum_{\alpha}\left|\dot{\gamma}^{\alpha}\right|=f \sum_{\alpha}\left|\dot{\gamma}^{\alpha, \mathrm{w}}\right|+(1-f) \sum_{\alpha}\left|\dot{\gamma}^{\alpha, \mathrm{c}}\right| .
$$


In this evolution law a decrease in the wall thickness with a saturation factor $k_{\mathrm{m}}$ is incorporated, with a final saturation value equal to $w_{\text {inf }}$, which is consistent with experimental observations (e.g. Fernandes and Schmitt (1983)).

The implementation of the model presented above follows an incremental-iterative solution procedure, which is applied for each of the composite components with the same imposed deformation (Taylor approach). The first step in this procedure is the initial estimate for the elastic part $\boldsymbol{F}_{\mathrm{e}}^{i}$, resulting in an estimate for the plastic part $\boldsymbol{F}_{\mathrm{p}}^{i}$ through (1). With the kinematics defined, both the stress and the Schmid stress is calculated. These values together with the slip resistance (8) (which is calculated from dislocation density evolution (11)) enter the slip law (7) resulting in the slip rates on each slip system. The updated plastic part of the deformation gradient is obtained from the calculated slip rates through a time integration scheme. Generally, the calculated and the imposed deformation will be different, which results in a residual. Iteration on the residual leads to updated values of variables including $\boldsymbol{F}_{\mathrm{p}}^{i}$ and $\boldsymbol{F}_{\mathrm{e}}^{i}$. With the current values of $r$ and $w$ the volume fraction $f$ is calculated with (10), which is used to determine the macroscopic stress (9). The procedure is repeated for all time steps, which results in the entire history of stress, slip and internal variable evolution.

\subsection{Orthogonal change in deformation}

An orthogonal change in the deformation path leads to dissolution of dislocation cell walls, which is captured through an increase in the wall thickness. In the limit the wall occupies the whole material where $w$ becomes equal to $r$, representing a full recovery of a uniform dislocation configuration (i.e. no cells present). This limit case is rarely observed in practice. After the dissolution process, new cells originate accommodating the new loading direction. Next, the cell size and dislocation density are considered to evolve in the same way as for monotonic deformation, i.e. the dislocation density increases in the walls and remains constant inside the cell. More experimental evidence is needed to improve further on this phenomenological relation.

The dissolution process is leading to a transient increase in the cell wall thickness driven by the overall slip rate as given by

$$
\dot{w}=k_{\mathrm{d}}(r-w) \sum_{\alpha}\left|\dot{\gamma}^{\alpha}\right|
$$

where $k_{\mathrm{d}}$ is the dissolution factor. The saturation value of the wall thickness logically equals the cell size $r$ corresponding to a complete dissolution of the cell. When both the dissolution and the redevelopment processes are taken into account, the wall evolution becomes

$$
\dot{w}=p k_{\mathrm{d}}(r-w) \sum_{\alpha}\left|\dot{\gamma}^{\alpha}\right|+(1-p) k_{\mathrm{m}}\left(w_{\mathrm{inf}}-w\right) \sum_{\alpha}\left|\dot{\gamma}^{\alpha}\right|
$$

where the first contribution on the right-hand side reflects equation (15) accounting for the effect of the loading in the new direction, i.e. the dissolution process. The second contribution on the right-hand side of (16) represents the development of the wall structure according to equation (14). The transition parameter $p$, to be specified in the following, defines the relative contribution of the dissolution process in the evolution of $w$. It is characterized by taking into account: (i) the dissolution process depends only on the angle between successive deformation paths and (ii) the dissolution effect disappears as the deformation proceeds in the new direction. In this context, the following expression for $p$ is taken:

$$
p=(1-|\theta|) \exp \left\{-B\left[\sum_{\alpha}\left|\gamma^{\alpha}\right|-\sum_{\alpha}\left|\gamma_{\text {pre }}^{\alpha}\right|\right]\right\},
$$


where $\theta$ is a scalar measure that identifies the strain path change and $\sum_{\alpha}\left|\gamma_{\text {pre }}^{\alpha}\right|$ indicates the accumulated plastic deformation prior to the strain path change and $B$ is a material parameter. To characterize the strain path change measure $\theta$, a commonly used definition is adopted here

$$
\theta=\frac{\boldsymbol{L}_{\mathrm{p} 1}: \boldsymbol{L}_{\mathrm{p} 2}}{\left|\boldsymbol{L}_{\mathrm{p} 1}\right|\left|\boldsymbol{L}_{\mathrm{p} 2}\right|}
$$

where $\boldsymbol{L}_{\mathrm{p} 1}$ and $\boldsymbol{L}_{\mathrm{p} 2}$ are the macroscopic plastic velocity gradient tensors prior to and after the strain path change. Here $\theta=1$ refers to monotonic deformation, $\theta=0$ to a cross test and $\theta=-1$ to a reverse test.

Equations (16) and (17) describe the evolution of the wall thickness during the whole deformation process. During monotonic deformation where $\theta=1$, equation (16) reduces to equation (14) describing cell wall thinning. After a strain path change, the dissolution is initiated with an intensity proportional to $(1-|\theta|)$. The dislocation walls start widening, governed by the competition between the new structure development and the old structure dissolution. As the deformation proceeds in the new direction, the dissolution process fades out and accordingly $p$ approaches 0 due to (17) and the wall thickness tends to decrease again: a new dislocation structure is developing.

\subsection{Reverse deformation}

The cell structure degeneration after a stress reversal, the so-called cell disruption, is modeled by a temporary increase in the cell size. As already discussed in section 2, the thickness of the cell walls does not change significantly due to stress reversal. Therefore, the other parameters, i.e. the wall thickness and the dislocation density, are assumed to evolve in a similar way as under monotonic deformation. The disruption of cells is observed to be a rapid process in which the size of the cells rapidly increases after a stress reversal and then slowly decreases (e.g. Viatkina et al (2003), Christodoulou et al (1986)). As the deformation proceeds in the opposite direction the cell size recovers to the level corresponding to the monotonic strain path (13). In order to model this temporary increase in the cell size, an additional (transient) term is incorporated in equation (13) for the cell size evolution:

$r=\frac{C G b}{f \sum_{\alpha} s_{a}^{\alpha, \mathrm{w}}+(1-f) \sum_{\alpha} s_{a}^{\alpha, \mathrm{c}}}+A \exp \left[-k_{\mathrm{c}}\left(\sum_{\alpha}\left|\gamma^{\alpha}\right|-\sum_{\alpha}\left|\gamma_{\mathrm{pre}}^{\alpha}\right|\right)\right]$,

where $A$ defines the degree of disruption and $k_{\mathrm{c}}$ is a constant reflecting the recovery speed (governed by slip). The entire term in the right-hand side (added to (13)) determines the immediate increase in the cell size. This effect vanishes with ongoing deformation depending on the value of the parameter $k_{\mathrm{c}}$. As soon as the disruption contribution gradually disappears, the cell size again follows the evolution as given for the monotonic deformation case. To reflect the fact that the cell disruption is triggered by a stress reversal, the coefficient $A$ depends on the strain path change through

$$
A= \begin{cases}a|\theta| & \text { if } \theta<0, \\ 0 & \text { if } \theta \geqslant 0,\end{cases}
$$

where $a$ is a fitting parameter. Consequently, the Bauschinger test triggers the highest disruption. For more complex strain path changes with negative values of $\theta$ both equations (16) and (19) have non-zero strain path change contributions ( $p>0$ and $A>0)$, describing a process with coexisting dissolution and disruption of cells. When $p=0$ and $A=0$, the model describes the evolution under monotonic deformation. In this way the resulting system of equations effectively unifies different types and combinations of strain path changes. 
Table 1. Material parameters for monotonic loading case. Some of the parameters, i.e. $\dot{\gamma}_{0}, G_{0}$ and $s$ have already been identified in previous work (Yalcinkaya et al 2008). The parameters $b$ and $G$ are taken from Frost and Ashby (1982). The value of $\rho_{0}$ is presented by Krejci and Lukas (1971). The latent interaction coefficient $A^{\alpha u}$ is identified and the self-interaction coefficient $A^{\alpha \alpha}$ is obtained by assuming a ratio of 1.4 between latent and self-hardening. The remaining parameters are identified by comparing the experimental trends with computational results. Additional parameters needed for complex strain paths are commented in the text.

\begin{tabular}{lll}
\hline Young's modulus & $E$ & $139 \mathrm{GPa}$ \\
Shear modulus & $G$ & $64 \mathrm{GPa}$ \\
Poisson's ratio & $v$ & 0.362 \\
Reference strain rate & $\dot{\gamma}_{0}$ & $1.07 \times 10^{6} \mathrm{~s}^{-1}$ \\
Burgers vector length & $b$ & $0.248 \times 10^{-9} \mathrm{~m}$ \\
Interaction coefficient (self) & $A^{\alpha \alpha}$ & 0.00072 \\
Interaction coefficient (latent) & $A^{\alpha u}$ & 0.001 \\
Initial dislocation density & $\rho_{0}$ & $0.18 \times 10^{14} \mathrm{~m}^{-2}$ \\
Activation free energy & $G_{0}$ & $2.95 \times 10^{-18} \mathrm{~J}$ \\
Dislocation multiplication parameter & $I$ & 0.228 \\
Dislocation annihilation rate parameter & $R$ & $5.1 \times 10^{-9}$ \\
Boltzmann constant & $k$ & $1.3807 \times 10^{-23} \mathrm{~J} \mathrm{~K}^{-1}$ \\
Thermal dislocation resistance & $s_{\mathrm{t}}$ & $15 \mathrm{MPa}$ \\
Saturation factor & $k_{\mathrm{m}}$ & 150 \\
Saturation value & $w_{\text {inf }}$ & $0.18 \times 10^{-6} \mathrm{~m}$ \\
Material constant & $C$ & 20 \\
\hline
\end{tabular}

\section{Numerical examples}

Using reported experimental trends, this section presents and qualitatively validates typical results: (i) the evolution of the internal variables during monotonic deformation and the intrinsic orientation effect during strain path changes of BCC single crystals and (ii) the macroscopic stress-strain behavior of BCC polycrystals during multi-stage loading processes. Due to the lack of quantitative experimental data, however, an adequate quantitative comparison is not possible, preventing a reliable quantification of the material parameters. Within a physically acceptable range of material parameters, it will be shown that the presented model is well capable of capturing all experimental trends. Young's modulus $E$, Poisson's ratio $v$, the reference strain rate $\dot{\gamma}_{0}$, the shear modulus $G$, the magnitude of the Burgers vector $b$, the interaction coefficients $A^{\alpha \alpha}$ and $A^{\alpha u}$, the activation energy $G_{0}$, thermal slip resistance $s_{\mathrm{t}}$, the dislocation multiplication parameter $I$ and the recovery length $R$ are the standard parameters in the constitutive model describing the BCC material, whereas $\rho_{0}, \rho_{\mathrm{c}}$, $k_{\mathrm{d}}, k_{\mathrm{m}}, w_{\text {inf }}, C, B, a$ and $k_{\mathrm{c}}$ are the additional parameters associated with the dislocation cell structure evolution. The set of standard parameters are well documented in the literature (see table 1). The remaining parameters have a restricted range and they are estimated to establish a qualitative agreement with experimental observations. The initial value of the dislocation densities in the walls $\rho_{0}$ logically equals $\rho_{\mathrm{c}}$ (no cells exist yet) and the initial value of the cell size $r_{0}$ can be calculated by using equation (13) where $f=1$ and $\rho_{\mathrm{w}}^{\alpha}=\rho_{0}^{\alpha}$.

\subsection{Example 1: monotonic deformation of single crystals}

Experimental observations of the microstructure evolution during monotonic deformation have been reported in section 2. In most cases, the length scales of the spatial patterns formed by the dislocations decrease with increasing strain. To analyze this change in the microstructure, several studies have been conducted. For instance, Sevillano et al (1981) present several 

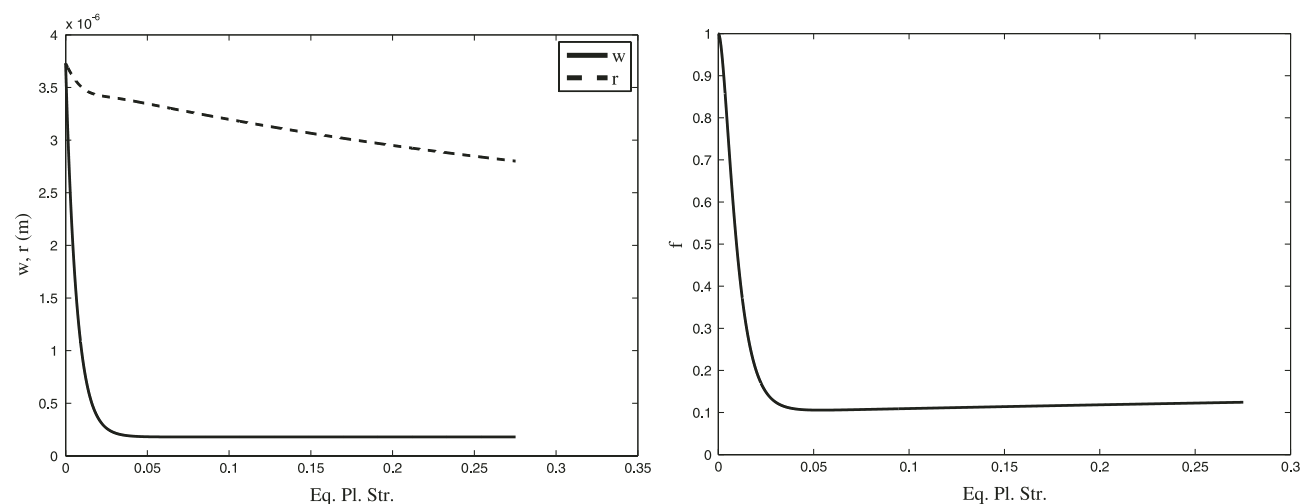

Figure 4. Evolution of the cell structure variables during monotonic deformation for $\alpha$-Fe. Left: wall thickness $w$ and cell size $r$. Right: volume fraction of the walls.

curves for the average cell size with respect to the deformation during rolling and drawing processes for different materials, and Fernandes and Schmitt (1983) give data on the wall thickness and dislocation cell size of low-carbon steel under various types of loading. In this framework, the parameters in the evolution equations are identified to retrieve this characteristic behavior.

In figure 4 the evolution of the microstructure of an $\alpha$-Fe single crystal during a uniaxial tension simulation at $298 \mathrm{~K}$ and a strain rate of $5 \times 10^{-4} \mathrm{~s}^{-1}$ is presented with the material parameters given in table $1^{3}$. The cell size shows a decreasing trend with increasing strain as expected. The wall thickness decreases quickly and stabilizes at a constant value. The volume fraction approaches a value of around 0.1 after a sharp decrease. This trend can also be found in the literature, where it is stated that the volume fraction of dislocation walls remains at a constant value (e.g. Peeters (2002)).

\subsection{Example 2: strain path change of single crystals}

In this example the effect of the intrinsic anisotropy of single BCC crystals during multi-stage loading is illustrated. In figure 5 the results of a cross loading simulation are presented where the crystal was first loaded in the [ $\left[\begin{array}{lll}0 & 0 & 1\end{array}\right]$ direction and next in the $\left[\begin{array}{lll}0 & 1 & 1\end{array}\right]$ direction after unloading at a strain value of 0.15 . For comparison purposes a reference calculation is performed in which the evolution of the microstructure is not incorporated, i.e. transient hardening and softening effects due to the strain path change (see solid line in figure 3) are absent. The dashed line presents the outcome of the full microstructure evolution computation, in which both the crystal slip anisotropy and the dislocation cell anisotropy are revealed. The purpose of this example is to discriminate these two intrinsic sources of anisotropy at the single crystal level. Obviously, both mechanisms here contribute to a larger yield stress after reloading. Whereas this increase is systematic for dislocation cell contribution, it is obviously orientation dependent for the slip anisotropy contribution.

${ }^{3}$ Note that the number of papers focusing on the determination of the interaction coefficients in BCC single crystals are very limited. Moreover, the actual value strongly depends on the actual BCC crystal considered and impurities present and the initial dislocation density. Values may therefore differ considerably, where a difference of a factor 10 can be easily found throughout the literature. 


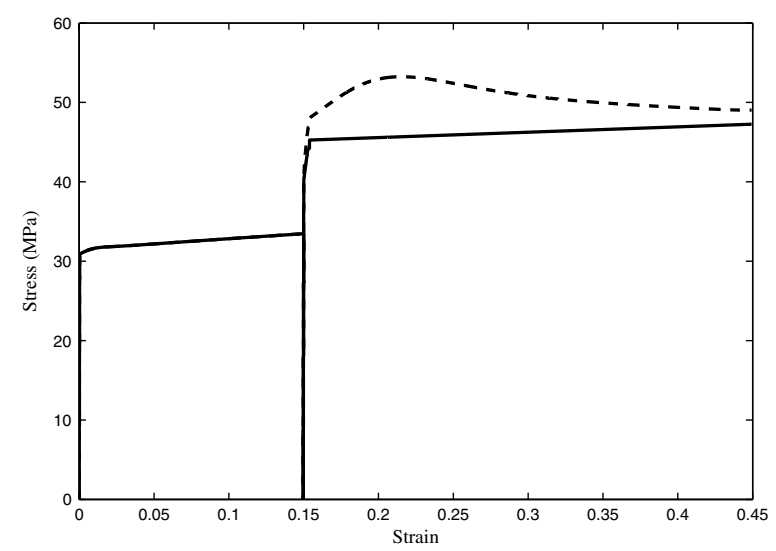

Figure 5. Stress-strain curve for the [ $\left[\begin{array}{lll}0 & 0 & 1\end{array}\right]$ uniaxial tension followed by the $\left[\begin{array}{lll}0 & 1 & 1\end{array}\right]$ uniaxial tension with (dashed line) and without (solid lines) microstructure evolution effect for $\alpha$-Fe single crystal.

\subsection{Example 3: strain path change of polycrystals}

In this subsection, the performance of the model in the context of complex deformation histories is evaluated by determining the response of a $\mathrm{BCC}$ polycrystal with a random texture under a sequence of: (i) two uniaxial tension tests in different directions to obtain the cross effect; (ii) simple shear and reversal in order to capture the Bauschinger effect.

As explained previously, at a single crystal level the effect of intrinsic crystallographic anisotropy during a strain path change is noticeably high. The initial anisotropy, the so-called orientation dependence of BCC single crystals has been studied before (e.g. Yalcinkaya et al (2008)) and this effect adds up to anisotropy due to the dislocation microstructure evolution. In this example the main interest focuses on the anisotropy due to substructure evolution during multi-stage loading processes for the case where the intrinsic orientation effect is known to contribute less. To this purpose polycrystal simulations have been conducted, where 100 randomly oriented crystals are considered interacting according to a Taylor averaging scheme.

First, the cell dissolution process and its macroscopic cross effect are analyzed. The characteristic feature of the stress-strain curve in figure 3 is the transient change induced by a change in the deformation path. An initial increase in the yield stress is followed by moderate softening. The cross effect vanishes gradually and the curve saturates towards to the monotonic case. In order to measure this effect experimentally either tension followed by simple shear or two successive orthogonal tensile experiments need to be conducted. With respect to the latter approach Schmitt et al (1991) presented clear experimental results where various tensile sequences were examined, with different angles between the succeeding tensile directions equal to $15^{\circ}, 45^{\circ}$ and $90^{\circ}$ with different amounts of prestrain. In Schmitt et al (1991), it was reported that no evolution of cell-blocks was observed, supporting the case examined here, where the cell structure development is assumed to be the main mechanism accompanying the strain path change. The sequence of two uniaxial tests with $45^{\circ}$ between the tensile axes is, according to equation (18), characterized by $\theta=0.25$, and it is rather close to a cross test exhibiting the highest cell dissolution. Additional to the parameters used during monotonic deformation (see table 1), $k_{\mathrm{d}}$ and $B$ are identified as 300 [-] and 20 [-] respectively. The obtained typical cross effect is presented in figure 6 . This transient effect is observed in many materials and the size of the effect is determined by the amount of applied prestrain while the shape of the hardening and softening zones depends on the material. 


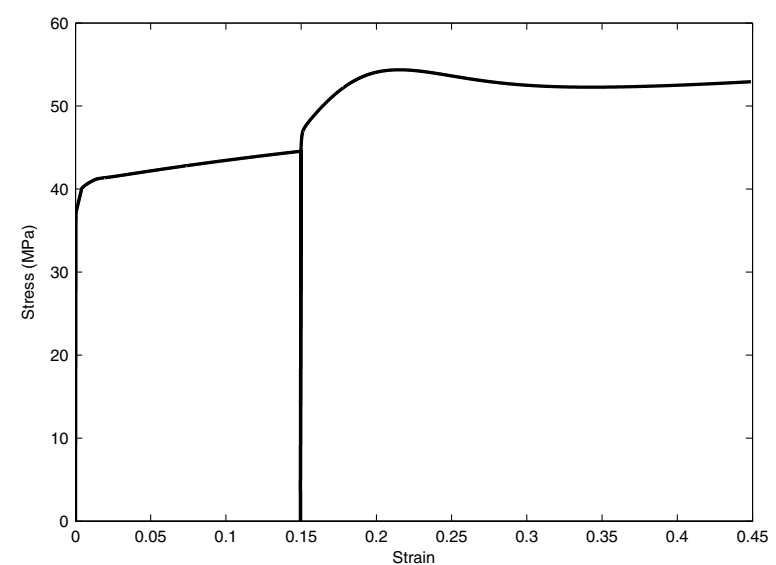

Figure 6. Predicted stress-strain curve of a $45^{\circ}$ cross tensile test of an $\alpha$-Fe polycrystal.

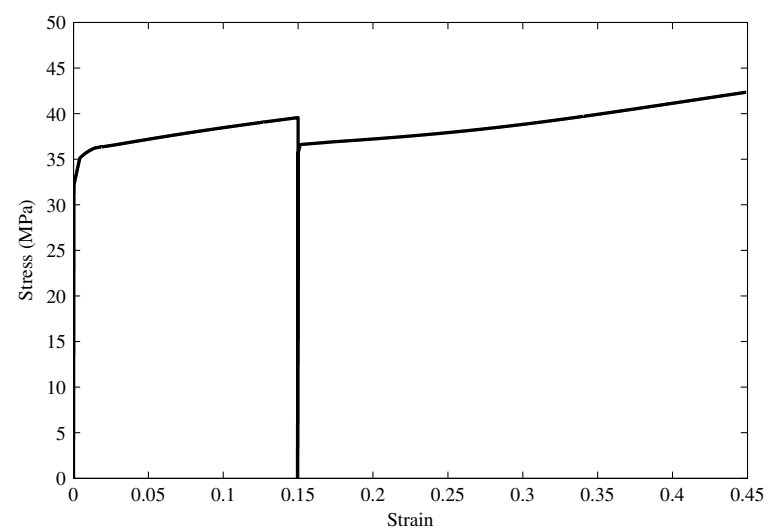

Figure 7. Bauschinger effect for shear-reverse shear test of an $\alpha$-Fe polycrystal.

The second example concerns the Bauschinger effect, which yields a reduction in the yield strength of the material after a load reversal. A simple shear and reversal simulation is presented in figure 7, where the evolution of the cell size $r$ is dominantly contributing to the anisotropy at this continuum level. Additional to the parameters used during monotonic deformation in table 1 , the parameters $a$ and $k_{\mathrm{c}}$ are identified as $1 \times 10^{-4} \mathrm{~m}$ and 14 [-] respectively, to validate this part of the model.

Even though both the cross effect and the Bauschinger effect are extensively documented in the literature, quantitative data on the evolution of dislocation cell structure during strain path changes remain hard to find. For this reason, a qualitative analysis rather than a quantitative study has been conducted here.

\section{Summary and conclusion}

This paper has presented a computational study on the anisotropy effects induced by strain path changes for BCC structured metals. For this purpose a composite dislocation cell model, which describes the dislocation substructure evolution, has been combined 
with a BCC crystal plasticity framework to bridge the dislocation cell structure evolution and its macroscopic anisotropic effects. The BCC crystal plasticity framework was based on Yalcinkaya et al (2008) and the composite cell model was built upon the contribution of Viatkina et al (2003), who analyzed strain path dependence phenomena in a phenomenological plasticity framework at small strains for FCC structured materials.

The presented computational framework assumed a composite aggregate, in which the material with a cell structure was considered to consist of two components: a soft cell interior component and hard cell wall components. The constitutive response of each component has been obtained from crystal plasticity simulations, while a set of phenomenological evolution equations for the cell size, the wall thickness and the dislocation density inside the walls captured the evolution of the microstructure.

The numerical examples of this work have revealed an adequate qualitative agreement between the simulations and the experimental trends for strain path change tests, i.e. a cross test and a Bauschinger test. Further quantitative analyses call for more extensive and more qualitative experimental results to compare with.

The paper clearly forwards a number of original contributions:

- A phenomenological cell structure evolution model embedded into a crystal plasticity framework is well able to reproduce all essential characteristics of strain path changes reported, consistently with experimental observations at two scales.

- The model proposed allows one to study the interaction between different sources of anisotropy, where a clear example at the single crystal and polycrystal has been given.

- The level at which the enrichment of the crystal plasticity model was made, enables its use in more complex microstructures as, e.g., multi-phase steels.

\section{Acknowledgments}

This research was carried out under the project number MC2.03158 in the framework of the Research Program of the Materials innovation institute M2i (www.m2i.nl), the former Netherlands Institute for Metals Research.

\section{References}

Bacroix B, Genevois P and Teodosiu C 1994 Eur. J. Mech. A/Solids 13 661-75

Bacroix B and Hu Z 1995 Metall. Mater. Trans. A 26 601-13

Barker I, Hansen N and Ralph B 1989 Mater. Sci. Eng. A $113449-54$

Barlat F, Ferreira Duarte J M, Gracio J J, Lopes A B and Rauch E F 2003 Int. J. Plast. $191215-44$

Bassani J L, Ito K and Vitek V 2001 Mater. Sci. Eng. A 319-321 97-101

Bouvier S, Gardey B, Haddadi H and Teodosiu C 2006 J. Mater. Process. Technol. 174 115-26

Christodoulou N, Woo O T and MacEwen S R 1986 Acta Metall. 34 1553-62

Duesbery M S and Vitek V 1998 Acta Mater. 46 1481-92

Fernandes J V and Schmitt J H 1983 Phil. Mag. A 48 841-70

Franciosi P and Zaoui A 1982 Acta Metall. 30 1627-37

Frost H J and Ashby M F 1982 Deformation Mechanism Maps: the Plasticity and Creep of Metals and Ceramics (Oxford: Pergamon)

Gardey B, Bouvier S, Richard V and Bacroix B 2005 Mater. Sci. Eng. A 400-401 136-41

Groma I 1997 Phys. Rev. B $565807-13$

Hansen N and Huang X 1997 Mater. Sci. Eng. A 234 602-5

Hill R and Rice J R 1972 J. Mech. Phys. Solids 20 401-13

Hoc T and Forest S 2001 Int. J. Plast. 17 65-85

Ito K and Vitek V 2001 Phil. Mag. 81 1387-407

Keh A S, Weissman S, Thomas G and Washburn J 1963 Electron Microscopy and Strength of Crystals (New York: Interscience) 
Krejci J and Lukas P 1971 Phys. Status Solidi a 5 315-25

Kuhlmann-Wilsdorf D 1962 Trans. Am. Inst. Min. Metall. Eng. 224 1047-61

Kuhlmann-Wilsdorf D 1989 Mater. Sci. Eng. A 113 1-41

Lee E H 1969 J. Appl. Mech. 36 1-6

Li Z J, Winther G and Hansen N 2006 Acta Mater. 54 401-10

Mollica F, Rajagopal K R and Srinivasa A R 2001 Int. J. Plast. 17 1119-46

Mughrabi H 1987 Mater. Sci. Eng. 85 15-31

Mughrabi H, Ungar T and Wilkens W 1986 Phil. Mag. A 53 793-813

Nesterova E V, Bacroix B and Teodosiu C 2001 Metall. Mater. Trans. A 32 2527-38

Peeters B 2002 PhD Thesis Katholieke Universiteit Leuven

Peeters B, Kalidindi S R, Van Houtte P and Aernoudt E 2000 Acta Mater. 48 2123-33

Queyreau S, Monnet G and Devincre B 2008 Int. J. Plast. 25 361-77

Rao B V N and Laukonis J V 1983 Mater. Sci. Eng. 60 125-35

Rauch E F 1991 Strength of Metals and Alloys Proc. ICSMA 9 (Haifa, Israel, 14-19 July 1991) vol 1, ed D G Brandon et al (London: Freund Publ. House, Ltd) pp 187-94

Rauch E F 1992 Solid State Phenom. 23-24 317-33

Rauch E F and Schmitt J H 1989 Mater. Sci. Eng. A 113 441-8

Rice J R 1971 J. Mech. Phys. Solids 19 433-55

Sang H and Lloyd D J 1979 Metall. Trans. A 10 1773-6

Schmitt J H, Fernandes J V, Gracio J J and F V M 1991 Mater. Sci. Eng. A 147 143-54

Sevillano J G, van Houtte P and Aernoudt E 1981 Prog. Mater Sci. 25

Tarigopula V, Hopperstad O S, Langseth M and H C A 2008 Eur. J. Mech. A/Solids 27 764-82

Teodosiu C 1992 Modeling of plastic deformation and its engineering applications Proc. 13th Ris $\phi$ Int. Symp. on Materials Science (Roskilde, Denmark) pp 125-46

Teodosiu C and Hu Z 1995 Simulation of materials processing: theory and applications Proc. NUMIFORM '95 (Balkema, Rotterdam) pp 173-82

Viatkina E 2005 PhD Thesis Eindhoven University of Technology

Viatkina E M, Brekelmans W A M and Geers M G D 2003 J. Phys. IV 105 355-62

Wagoner R H and Laukonis J V 1983 Metall. Trans. A 14 1487-95

Wang J, Levkovitch V, Reusch F, Svendsen B, Huetink J and van Riel M 2008 Int. J. Plast 24 1039-70

Wilson D V and Bate P S 1994 Acta Metall. Mater. 42 1099-111

Yalcinkaya T, Brekelmans W A M and Geers M G D 2008 Modell. Simul. Mater. Sci. Eng. 16085007 